\title{
Implementation of a Deutsch-like quantum algorithm utilizing entanglement at the two-qubit level on an NMR quantum-information processor
}

\author{
Kavita Dorai, ${ }^{1, *}$ Arvind, ${ }^{3, \dagger}$ and Anil Kumar ${ }^{1,2, \ddagger}$ \\ ${ }^{1}$ Department of Physics, Indian Institute of Science, Bangalore 560012, India \\ ${ }^{2}$ Sophisticated Instruments Facility, Indian Institute of Science, Bangalore 560012, India \\ ${ }^{3}$ Department of Physics, Guru Nanak Dev University, Amritsar 143005, India
}

\begin{abstract}
We describe the NMR implementation of a recently proposed quantum algorithm involving quantum entanglement at the level of two qubits. The algorithm solves a generalization of the Deutsch problem, and distinguishes between even and odd functions using fewer function calls than is possible classically. The manipulation of entangled states of the two qubits is essential here, unlike the Deutsch-Jozsa algorithm and Grover's search algorithm for two bits.
\end{abstract}

It was demonstrated recently that a quantum computer, exploiting quantum state superposition and entanglement, is definitely more powerful than any existing classical computer [1-5]. NMR has been most successful as a testbed for ideas in quantum computing, despite apprehensions [6,7]. The visualization of a spin-1/2 particle as a qubit, combined with existing multidimensional NMR methods, has led to breakthroughs in pseudopure state preparation [8-12], the demonstration of universal quantum logic gates [13-17], and the implementation of various quantum algorithms for up to five qubits [18-29].

Early on, Deutsch and Jozsa presented a quantum algorithm to determine whether a Boolean function $f$ is constant or balanced [2] (also see Ref. [3]). Recently, the DeutschJozsa (DJ) algorithm was modified and implemented using a lesser number of input qubits [30-32], and interest continues in further generalizations of the DJ problem [33]. The DJ algorithm allows a classical description for the two-qubit case, and it is only for three or more qubits that the quantum nature of the algorithm is displayed [30,31]. As pointed out by Lloyd [34], Grover's search algorithm for two qubits also does not require entangling transformations.

A quantum algorithm to distinguish between even and odd functions was recently designed [35]. The algorithm uses entangling transformations at the two-qubit level itself, and is an interesting example of the power of a quantum computer over corresponding classical systems. In this paper, we present the experimental implementation of the algorithm on a two-qubit NMR quantum computer. The requisite unitary transformations were implemented using spin-selective and composite- $z$ pulses. A judicious combination of composite pulses and evolution under the scalar coupling Hamiltonian has been used to construct the desired entangling transformations. The algorithm requires one to distinguish between nonorthogonal states of the two qubits in order to classify the functions [36]. In our NMR implementation, such a distinction is achieved in a single measurement.

\footnotetext{
*Email address: kavita@physics.iisc.ernet.in

${ }^{\dagger}$ Email address: arvind@physics.iisc.ernet.in

*Email address: anilnmr@physics.iisc.ernet.in
}

Consider a Boolean function defined from a two-bit domain space to a one-bit range space: $f(x):\{0,1\}^{2} \rightarrow\{0,1\}$. There are four possible input values $(00),(01),(10)$, and (11), the output for each of these being either 0 or 1 . The 16 possible functions can be divided into subclasses based on the number of ones and zeros in their outputs. The functions can be categorized in the subclasses [0,4], [1,3], $[2,2],[3,1]$, or $[4,0]$, where the first entry indicates the number of ones and the second indicates the number of zeros in the output. The functions with an even number of ones in the output (the functions belonging to the categories $[0,4],[2,2]$ and $[4,0])$ are denoted "even" functions, while the functions with an odd number of ones in the output (the $[1,3]$ and $[3,1]$ functions) are denoted "odd" functions. For the two-qubit case, we thus have eight even and eight odd functions.

Classically, the classification of a function as even or odd would require computing it at all input points. The quantum algorithm constructed uses just two function calls to evaluate the even or odd character of the given function [35]. The function call mechanism is similar to the one used to solve the modified Deutsch problem [30,31]. Each function $f$ can be encoded by a unitary transformation $U_{f}$, with its action on the eigenstates of the two qubits defined as

$$
U_{f}=\left(\begin{array}{cccc}
(-1)^{f(00)} & 0 & 0 & 0 \\
0 & (-1)^{f(01)} & 0 & 0 \\
0 & 0 & (-1)^{f(10)} & 0 \\
0 & 0 & 0 & (-1)^{f(11)}
\end{array}\right)
$$

Pseudo-Hadamard gates [15] are practically equivalent to the Hadamard operator, and convert an eigenstate to a uniform superposition of all possible eigenstates. The one-qubit pseudo-Hadamard gate $h$ is given by 


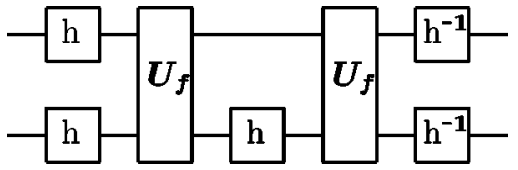

FIG. 1. Quantum circuit for two qubits, that implements the algorithm to distinguish between even and odd functions, using pseudo-Hadamard and inverse pseudo-Hadamard gates (denoted by $h$ and $h^{-1}$, respectively) and $U_{f}$ transformations.

$$
\begin{aligned}
& |0\rangle \rightarrow \frac{h}{\sqrt{2}}(|1\rangle+|0\rangle) \\
& |1\rangle \rightarrow \frac{h}{\sqrt{2}}(|1\rangle-|0\rangle)
\end{aligned} ; \quad h=\frac{1}{\sqrt{2}}\left(\begin{array}{ll}
1 & 1 \\
-1 & 1
\end{array}\right)
$$

The pseudo-Hadamard gate has been applied on both qubits nonselectively, as well as selectively on one qubit alone. The corresponding gates are denoted as

$$
h^{(1)}=h \otimes I ; \quad h^{(2)}=I \otimes h ; \quad h^{(1,2)}=h \otimes h,
$$

where 1 and 2 label the qubit involved.

There are sixteen $U_{f}$ matrices in all, with eight of them being entangling and the rest nonentangling in character. For example, the $U_{f}$ matrix with diagonal entries $[1,1,1,-1]$ cannot be written as a tensor product of two matrices, one belonging to each qubit. This unitary transformation is hence entangling in nature. It is interesting to note that for the two-bit case, the subclass of functions that are either constant or balanced in the sense of the Deutsch problem, i.e., the functions belonging to the $(0,4)$ and $(2,2)$ subclasses, are all separable in character. Therefore, the two-bit Deutsch problem affords a classical explanation, and can be implemented using nonentangling transformations alone.

A quantum circuit to evaluate the even or odd nature of a function is shown in Fig. 1. The algorithm starts with both qubits in a thermal (mixed) initial state. A pseudo-Hadamard transformation is applied on both qubits, resulting in both of them being in a state which is a superposition of all possible input states. The desired unitary transformation $U_{f}$ is then applied on this superposition state, followed by a pseudoHadamard gate operating on the second qubit alone. The function is called again through $U_{f}$, and an inverse pseudoHadamard is applied on both qubits before the readout operation.

The result of the computation is encoded in the final state of the two qubits. If the function is even, both the qubits are in an unentangled state and a signal is obtained for the qubit on which the selective pseudo-Hadamard gate is applied, with no signal being obtained for the other qubit. If the function is odd, both qubits are in an entangled state, corresponding to multiple-quantum coherence of the two spins, and no observable signal is obtained. The even or odd nature of the function can thus be distinguished "pictorially,' by looking at the final NMR spectrum.

Previously, the algorithm has been described for a pure initial state [35]. In NMR one normally encounters mixed states. Hence we first generalize the procedure to include

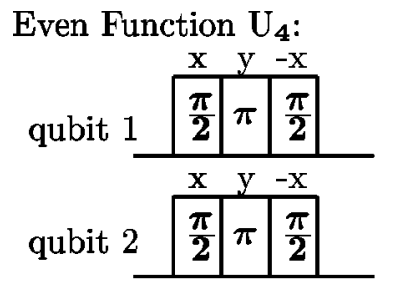

Odd Function $\mathrm{U}_{\boldsymbol{9}}$ :

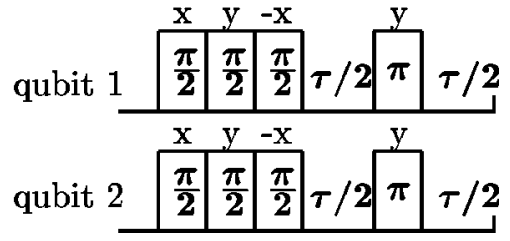

FIG. 2. NMR pulse sequences to implement the even function $U_{4}$ and the odd function $U_{9}$. The pulses are represented by boxes, with the phase of each pulse written above it. Composite- $z$ pulses are implemented by three pulses, applied back to back. The time period $\tau$ is set to $1 / 2 J, J$ being the value of the spin-spin coupling. The pulse schemes for the other functions are similar, and can be constructed by varying the phase of the composite- $z$ pulses.

mixed initial states. Consider the two qubits in an initial state described by the deviation density matrix

$$
\Delta \rho_{\text {initial }}=\left(\begin{array}{cccc}
k_{1} & 0 & 0 & 0 \\
0 & 0 & 0 & 0 \\
0 & 0 & 0 & 0 \\
0 & 0 & 0 & k_{2}
\end{array}\right)
$$

with $k_{1}$ and $k_{2}$ being independent variables. For $k_{1}=1$ and $k_{2}=0$ this density matrix represents a pseudopure state. For $k_{1}=1$ and $k_{2}=-1$ it represents a thermal state. Multiples of identity have been ignored in the density matrix, as they do not contribute to the measured signal in an NMR experiment.

We now evolve this density matrix through the sequence of operations $h^{(1,2)} U_{f} h^{(2)} U_{f}\left[h^{(1,2)}\right]^{-1}$. After some algebra, this leads to the deviation density matrixes

$$
\begin{gathered}
\Delta \rho_{\text {even }}=\frac{1}{2}\left(\begin{array}{cccc}
k_{2} & \zeta k_{2} & 0 & 0 \\
\zeta k_{2} & k_{2} & 0 & 0 \\
0 & 0 & k_{1} & \zeta k_{1} \\
0 & 0 & \zeta k_{1} & k_{1}
\end{array}\right), \\
\Delta \rho_{\text {odd }}=\frac{1}{2}\left(\begin{array}{cccc}
k_{1}+k_{2} & 0 & 0 & \zeta\left(k_{1}-k_{2}\right) \\
0 & 0 & 0 & 0 \\
0 & 0 & 0 & 0 \\
\zeta\left(k_{1}-k_{2}\right) & 0 & 0 & k_{1}+k_{2}
\end{array}\right),
\end{gathered}
$$

where $\zeta=+1$ or -1 , for different $U_{f}$ transformations. For $k_{1}=1$ and $k_{2}=0$ we arrive at the pure state result of Ref. [35] i.e., the final state is $|00\rangle+\zeta|01\rangle$ for an even function and $|00\rangle+\zeta|11\rangle$ for an odd function. For a thermal initial state, i.e., $k_{1}=-k_{2}=1$, the deviation density matrices $\Delta \rho_{\text {even }}$ and $\Delta \rho_{\text {odd }}$, can be distinguished unambiguously by a 

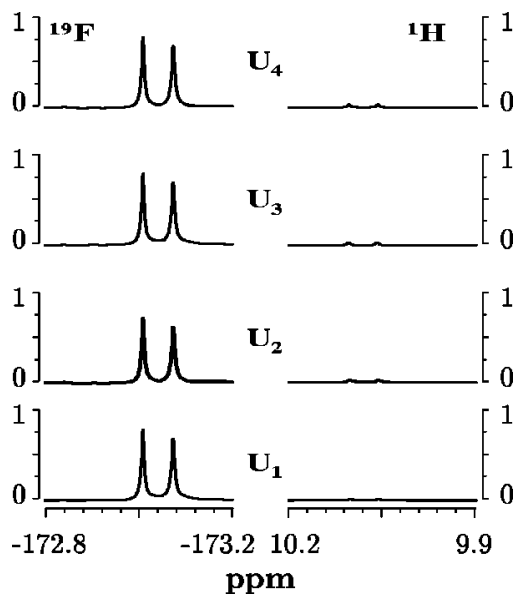

FIG. 3. The implementation of the even transformations $U_{1}$ $-U_{4}$ on the two qubits $\left({ }^{19} \mathrm{~F}\right.$ and $\left.{ }^{1} \mathrm{H}\right)$ of 5 -fluorouracil. The corresponding unitary matrices have entries along the diagonals of $[1,1,1,1],[1,1,-1,-1],[1,-1,1,-1]$, and $[1,-1,-1,1]$ respectively. The transformations $U_{5}-U_{8}\left(U_{i+4}=-U_{i}, i=1,2,3\right.$, and 4), lead to exactly the same results (spectra not shown). All spectra have been plotted to the same scale.

single NMR measurement. The spectrum for even functions will give two lines corresponding to the observable singlequantum coherences present in the density matrix. For odd functions there is no signal, as the only nondiagonal elements of the density matrix are those corresponding to double-quantum coherences. We note here that a single NMR measurement is able to distinguish between two nonorthogonal quantum states, which is normally not possible using other measurement techniques [36]. We further note that the presence of double-quantum coherences in the final state for the odd functions shows the entangling nature of the unitary transformations used. The use of a Hadamard instead of a pseudo-Hadamard transformation would lead to results which are qualitatively similar. The entanglement in the final state would show up as a zero-quantum coherence of the two qubits, instead of a double-quantum coherence [35].

We have implemented the two-qubit even-odd quantum algorithm on an NMR quantum computer using the molecule 5-fluorouracil dissolved in dimethyl sulfoxide. This fluorineproton spin system is a good candidate for quantum computing since it has good sensitivity, a resolved $J$ coupling of 6.1 $\mathrm{Hz}$, and the duration of spin-selective pulses can be relatively short. All experiments were performed on a Bruker AMX-400 spectrometer at room temperature. The pseudoHadamard gate was achieved by applying a $\left(90^{\circ}\right)_{y}$ pulse selectively on a spin, or nonselectively on both spins, as the case maybe. Pulse schemes consisting of sandwiches of composite and spin-selective pulses (described schematically in Fig. 2) are used to implement the even and odd $U_{f}$ transformations.

As an illustration, the unitary transformation $U_{4}$ (given by the diagonal matrix $[1,-1,-1,1]$ ) corresponds to a $\pi$ rotation about the $z$ axis of both the qubits, up to a global phase factor. Global phase changes are not detectable in NMR, and are hence ignored. The $z$ rotation can be implemented using a composite-pulse sandwich, as a set of rotations about the $x$

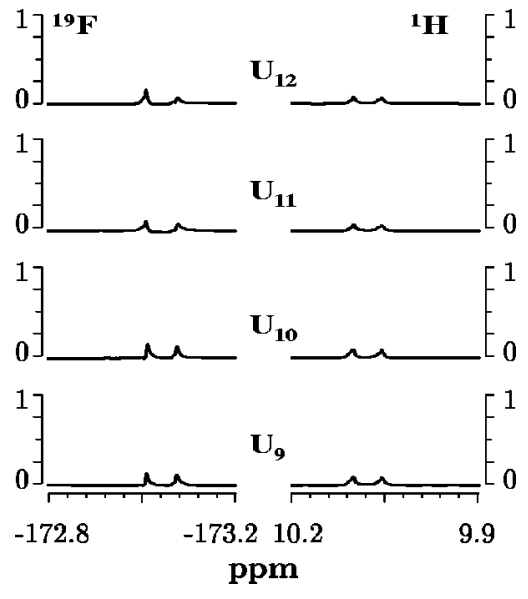

FIG. 4. The implementation of odd functions $U_{9}-U_{12}$ on 5-fluorouracil, using entangling transformations. The absence of all spectral lines indicates the odd nature of the $U_{f}$ 's. The corresponding unitary matrices have entries along the diagonal of $[1,-1,-1,-1],[-1,1,-1,-1],[-1,-1,1,-1]$, and $[-1,-1,-1,1]$, respectively. The odd functions $U_{13}-U_{16}\left(U_{i+4}=U_{i}, i=9,10,11\right.$, and 12) lead to the same results (spectra not shown). A $\tau$ period of $82.24 \mathrm{~ms}$ has been used to implement the evolution under the scalar coupling Hamiltonian. All the spectra shown have been plotted to the same scale as in Fig. 3.

and $y$ axes $[\theta]_{z} \equiv[\pi / 2]_{x}[\theta]_{y}[\pi / 2]_{-x}$. The $U_{f}$ 's encoding the other even functions $U_{1}, U_{2}$, and $U_{3}$ correspond to the do-nothing operation (the transformation is the unity matrix), a $[\pi]_{z}$ rotation in the single-spin subspace of the first qubit, and $\mathrm{a}[\pi]_{z}$ rotation on the second qubit, respectively, and have been constructed using similar pulse schemes. All these even transformations can be decomposed into transformations in the subspaces of each individual qubit, and have hence been implemented experimentally without invoking quantum entanglement. The result of applying all the even transformations on the two qubits is shown in Fig. 3. Spinselective pulses of $12.7 \mu \mathrm{sec}$ on the proton and $22.1 \mu \mathrm{sec}$ on the fluorine have been used to achieve good selectivity. The spectra obtained reveal a retention of the fluorine spin single-quantum coherence, while no lines are seen for the proton (whose coherence was converted back to unobservable $z$ magnetization by the selective pseudo-Hadamard gate).

We have used entangling transformations to implement the odd functions. Consider the nonseparable $U_{9}$ matrix with the entries $[1,-1,-1,-1]$ along its diagonal. The transformation is achieved experimentally by a $[\pi / 2]_{z}$ rotation on the first spin, followed by a $[\pi / 2]_{z}$ rotation on the second spin, and then a free evolution for a time interval $\tau$ tailored to $\tau=1 / 2 J, J$ being the value of the scalar coupling. A nonselective $\pi$ pulse has been applied in the middle of the $\tau$ interval, to refocus the chemical shift evolution. The $[\pi / 2]_{z}$ rotations in the single-spin subspaces have been achieved by the composite pulse sandwiches $[\pi / 2]_{x}[\pi / 2]_{y}[\pi / 2]_{-x}$, selective on the spin concerned. The other odd functions have been similarly implemented. The spectra corresponding to the experimental implementation of the odd functions are 
shown in Fig. 4. The states of the two qubits are completely entangled, leading to no observable spectral lines for all these functions. All the spectra in both Figs. 3 and 4 have been plotted to the same scale.

In conclusion, we have shown the NMR implementation of a quantum algorithm that uses the entangled states of two input qubits to distinguish between even and odd functions. We have used spin-selective composite pulse sandwiches and evolution under scalar coupling to implement the required nontrivial entangling transformations. It is to be noted that NMR experiments are able to distinguish between two nonorthogonal quantum mechanical states in a single measurement.

SIF at IISc Bangalore is acknowledged for the use of the AMX-400 spectrometer and IISc for financial support.
[1] D.P. DiVincenzo, Science 270, 255 (1995); I.L. Chuang, R. Laflamme, P.W. Shor, and W.H. Zurek, ibid. 270, 1633 (1995); S. Lloyd, ibid. 273, 140 (1996); A. Steane, Rep. Prog. Phys. 61, 117 (1998); J. Preskill, Proc. R. Soc. London, Ser. A 454, 385 (1998).

[2] D. Deutsch and R. Jozsa, Proc. R. Soc. London, Ser. A 439, 553 (1992).

[3] R. Cleve, A. Ekert, C. Macchiavello, and M. Mosca, Proc. R. Soc. London, Ser. A 454, 339 (1998).

[4] P.W. Shor, SIAM J. Comput. 26, 1484 (1997).

[5] L.K. Grover, Phys. Rev. Lett. 79, 325 (1997).

[6] S.L. Braunstein, C.M. Caves, R. Jozsa, N. Linden, S. Popescu, and R. Schack, Phys. Rev. Lett. 83, 1054 (1999).

[7] N. Linden and S. Popescu, LANL e-print quant-ph/9906008.

[8] N. Gershenfeld and I.L. Chuang, Science 275, 350 (1997).

[9] D.G. Cory, A.F. Fahmy, and T.F. Havel, Proc. Natl. Acad. Sci. U.S.A. 94, 1634 (1997).

[10] E. Knill, I.L. Chuang, and R. Laflamme, Phys. Rev. A 57, 3348 (1998).

[11] Lieven. M.K. Vandersypen, C.S. Yannoni, M.H. Sherwood, and I.L. Chuang, Phys. Rev. Lett. 83, 3085 (1999).

[12] Kavita Dorai, Arvind, and Anil Kumar, Phys. Rev. A 61, 042306 (2000).

[13] D.G. Cory, M.D. Price, and T.F. Havel, Physica D 120, 82 (1998).

[14] M.A. Nielsen, E. Knill, and R. Laflamme, Nature (London) 396, 52 (1998).

[15] J. A. Jones, R. H. Hansen, and M. Mosca, J. Magn. Reson. 135, 353 (1998).

[16] Z.L. Madi, R. Bruschweiler, and R.R. Ernst, J. Chem. Phys. 109, 10603 (1998).

[17] J. A. Jones and E. Knill, J. Magn. Reson. 141, 322 (1999).
[18] J.A. Jones and M. Mosca, J. Chem. Phys. 109, 1648 (1998).

[19] I.L. Chuang, L.M.K. Vandersypen, X. Zhou, D.W. Leung, and S. Lloyd, Nature (London) 393, 143 (1998).

[20] I.L. Chuang, N. Gershenfeld, and M. Kubinec, Phys. Rev. Lett. 80, 3408 (1998).

[21] J.A. Jones, M. Mosca, and R.H. Hansen, Nature (London) 393, 344 (1998).

[22] J.A. Jones, Science 280, 229 (1998).

[23] N. Linden, H. Barjat, and R. Freeman, Chem. Phys. Lett. 296, 61 (1998).

[24] R. Marx, A.F. Fahmy, J.M. Myers, W. Bermel, and S.J. Glaser, LANL e-print quant-ph/9905087.

[25] J.A. Jones and M. Mosca, Phys. Rev. Lett. 83, 1050 (1999).

[26] R. Nelson, S. Lloyd, and D.G. Cory, Phys. Rev. A 61, 022106 (2000).

[27] D.G. Cory, W. Maas, M. Price, E. Knill, R. Laflamme, W.H. Zurek, T.F. Havel, and S.S. Somaroo, Phys. Rev. Lett. 81, 2152 (1998).

[28] C.H. Tseng, S. Somaroo, Y. Sharf, E. Knill, R. Laflamme, T.F. Havel, and D.G. Cory, Phys. Rev. A 61, 012302 (2000).

[29] T. S. Mahesh, Kavita Dorai, Arvind, and Anil Kumar, J. Magn. Reson. 148, 95 (2001).

[30] D. Collins, K.W. Kim, and W.C. Holton, Phys. Rev. A 58, R1633 (1998).

[31] Arvind, Kavita Dorai, and Anil Kumar, LANL e-print quant-ph/9909067.

[32] Jaehyun Kim, Jae-Seung Lee, Soonchil Lee, and Chaejoon Cheong, LANL e-print quant-ph/9910015.

[33] D.P. Chi, J. Kim, and S. Lee, LANL e-print quant-ph/0005059.

[34] Seth Lloyd, Phys. Rev. A 61, 010301 (2000).

[35] Arvind and N. Mukunda, LANL e-print quant-ph/0006069.

[36] B. Huttner, A. Muller, J.D. Gautier, H. Zbinden, and N. Gisin, Phys. Rev. A 54, 3783 (1996). 